Ausbeute an Gallussäure wurde mit 20 prozentiger Schwefelsäure erhalten. Woraus der schwarzbraune Niederschlag besteht, und was sich aus dem Rest der Chebulinsäure gebildet hat, ist mir zur Zeit nicht möglich zu sagen. Wird die saure wässerige Lösung, nach Entfernung der Gallussäure, vom gelösten Äther befreit und mit Essigäther ausgeschüttelt, so hinterläfst dieser beim Verdunsten nur einen amorphen Rückstand.

Die Reduktionsversuche, welche ich mit der Chebulinsäure ausführte, waren, aus Mangel an Material, nicht von dem gewünschten Erfolge bogleitet. Auch bei der Reduktion der Gallussäure und des Tannins konnte ich Benzoësüure und Salicylsäure, welche nach den Angaben von Guignet (Compt. rend. 113, 200) entstehen sollen, richt mit Sicherheit nachweisen.

\title{
Bestimmung des Eisens im Brunnenwasser auf kolorimetrischem Wege und Verwendung der Gerbsïure zu diesem Zwecke
}

\author{
von Dr. F. Gerhard in Wolfenbiittel.
}

Eingegangen den 10. XII. 1892.

Das Vorkommen von Eisen in Trinkwässern hat seit einigen Jahren eine erhöhte Bedeutung gewonnen. Während es nämlich in früheren Zeiten meist üblich war, für städtische Wasserleitungen das Wasser der nahen Flüsse zu verwenden, kommt man hiervon mehr zurück und sucht das nötige Wasser dem Untergrunde mittels Tiefbrunnen zu entnehmen. Solches Wasser ist in der Tat häufig auch sehr rein und gut, und ein bei weitem besseres Trinkwasser. als das der Flüsse.

Nun macht man aber häufig die Frfahrung, dal's das Wasser solcher Tiefbrunnen sich eisenhaltig zeigt. Sehr bekannt ist dies in letzter Zeit bei dem Bohren von Brunnen in der Stadt Hamburg geworden, wo die meisten derselben ein eisenhaltiges Wasser lieferten. Und auch in anderen Orten, besonders der norddeutschen Tief-Ebene hat man diese Beobachtung gemacht. So kamen denn die Analytiker 
öfters in die Lage, Wasser auf den Eisengehalt zu prüfen und die Menge des Eisens zu bestimmen.

Für praktische Zwecke wird nun das Eisen gewöhnlich auf kolorimetrischem Wege ermittelt. Nach Kubel und Tiemann wird ein Quantum Wasser, mit etwas Salzsäure und Kaliumchlorat versetzt, eingeengt und in dieser (von Chlor sorgfältig befreiten) Flülsigkeit die Menge des Eisens durch Zufügung von Rhodankalium oder Ferrocyankalium bestimmt. Die Methode ist nicht eben bequem, es mul's ein Eindampfen stattfinden und dann das überschülsige Chlor entfernt werden, und das ist nicht so sehr schnell auszuführen. Hinsichtlich der Frage, welchem Reagens der Vorzug zu geben sei, sind die Meinungen geteilt. Kubel und Tiemann empfehlen das Blutlaugensalz, Jolles und Proskauer bevorzugen das Rhodankalium. Fis ist sicher. dals sich bei Beiden kleine Mifsstände bemerkbar machen, und dals es einiger Uebung bedarf, um gleichmäfsige, gut vergleichbare Färbungen zu erzielen.

Beim Rhodankalium ist zu bemerken, dafs dasselbe nicht selur empfindlich ist, bei weitem nicht so empfindlich als Ferrocyankalium. Für das erstere Reagens liegt die Empfindlichkeitsgrenze bereits bei $0 . \tilde{y}$ Milligu. Fe in Liter, schon 0.2 Milligr. Fe giebt keine irgend bemerkbare Fürbung mehr.

Ferrocyankalium ist schärfer, mit 0,5 Milligr. Fe im Liter erlält man eine schöne, hellblaue Farbe und selbst 1/10 Milligr. giebt noch eine bemerkbare, allerdings sehr schwache Fürbung. Die Reaktion ist so empfindlich, dals man bei Anwendung dieses Reagenzes nur selten nötig haben wird, das zu untersuchende Wasser aut ein geringes Volumen einznengen.

Auch die Ueberfübrung des Eisenoxyduls in Oxyd durch Erhitzen mit Salzsiaure und Kaliumchlorat kann man meistens umgehen. Das Eisen ist in den Brunnenwässern ursprünglich wohl stets als Ferrokarbonat gelöst; das Wasser ist klar und hat einen tintenartigen Geschmack. Aber schon nach kurzer Zeit beginnt es sich zu trüben in Folge der Ueberführung des Karbonates in Hydroxyd durch den Sauerstoff der Iuft, und nach wenigen Stunden ist in dem Wasser keine Spur von. Eisen mehr in Tiïsung, alles Eisen liegt als Hydroxyd am Boden. 
Versetzt man das Wasser nun mit etwas Salzsäure und erwärmt, so erhält man anf dis einfachste Weise eine zur kolorimetrischen Bestimmung geeignete Flüssigkeit, die alles Eisen als Chlorid enthält, und in der dasselbe bis auf $1 / 5 \mathrm{mg}$ im Liter genau ermittelt werden kann. Will man noch geringere Mengen feststellen, so mufs man allerdings einengen, doch wirà dies für praktische Zwecke wohl seltener nötig sein. Wie oben erwähnt, verrät sich selbst $1 / 10 \mathrm{mg}$ im Liter noch durch einen schwach bläulichen Schimmer. Ein so geringer Gehalt an Eisen verrät sich dem uufmerksamen Kenner allerdings immer noch durch Aussehen und Geschmack des Wassers, vom Publikum wird es aber nicht bemerkt. Ich will hier erwähnen, dals nach den Beschlüssen des 6. internationalen Pharm.-Kongresses in Brüssel im Trinkwasser nicht mehr als $3 \mathrm{mg}$ Eisen im Liter enthalten sein soll. Meiner Meinung nach ist diese Grenze zu hoch. Wenn ein solcher Gehalt an Eisen auch nicht gesundheitsschädlich wirkt, so kann ein dorartiges Wasser doch nicht als ein normales Trinkwasser gelten.

Wenn wir nun auch im Rhodankalium und Ferrocyankalium gute Reagentien auf Eisen besitzen und insonderheit das letztere sich als aufserordentlich empfindlich erweist, so giebt es doch noch ein anderes Reagens, das, wie mir scheint, noch etwas empfindlicher und auch sonst den beiden vorzuziehen ist. Dieses Reagens ist die Gerbsäure. Die Verwendung derselben gewährt folgende Vorzüge:

1. Sie ist ebenso empfindlich und vielleicht sogar noch etwas empfindlicher als Ferrocyankalium, und man hat infolge dessen selten nötig, das zu untersuchende Wasser zu konzentrieren.

2. Die Reaktion in Wässern tritt auch ein, in denen sich das Eisen noch auf der Oxydulstufe befindet; es ist eine vorherige Überführung des Eisens in Oxydsalz nicht nötig.

3. Die Färbung tritt schneller, gleichmälsiger und sicherer ein, als bei den andern beiden Reagentien.

Von den verschiedenen Arten von Gerbsäure (Katechu-, China-, Kaffee-, Ratanhia-Gerbsäure) hat sich mir die Gallusgerbsäure (das Tannin der Apotheken) als das brauchbarste erwiesen.

Um nun aber die Gerbsäure zur kolorimetrischen Prüfung verwenden zu kïnnen, mufs man selbstverständlich in solcher Weise arbeiten, dafs man stets klare Flüssigkeiten erhält, sonst sind rich- 
tige Vergleichungen unmöglich. Versetzt man eisenhaltiges Wasser ohne weiteres mit Gerbsäure. so entstehen Trübungen und milsfarbige Niederschläge, Realtionen, die für den vorliegenden Zweck absolut nicht zu verwerten sind.

Bietet man jedoch das Eisen zudem Tannin in schwach alkalischer Lösung oder in einer Form, wo es durch Alkalien vicht gefällt werden würde, so entsteht wicht ein Niederschlag, sondern nur eine intensive, schöne rosa otter lila Färbung.

Bekanntlich yerden weinsaures, citronensaures Eisenoxyd, EisenDoppelsalze der Pyrophosphorsäure u. a. durch Alkalien nicht gefiallt, und diese sind daher geeignet zur Anstellung der Reaktion. Von den genannten Salzen habe ich das letztgenannte (Salz der Pyrophosphorsäure) für das geeignetste befunden. Um die Reaction mit Tannin anzustellen, versetzt man deshalb das Wasser zunächst mit einer Lösung von krystall. Natriumpyrophosphat und fügt erst dann das Reagens zu.

Beim Versetzen einer Kalk und Eisen etc. enthaltenden Flüssigkeit mit Natriumpyrophosphat entsteht zuerst ein Niederschlag von Calcium- und Eisenpyrophosphat; derselbe löst sich jedoch in einem Überschusse des angewandten Salzes auf, und man erhält sofort eine klare Lösung. Fügt man nun Tannin zu, so entsteht eine schöne rosa oder lila Fürbung, die bei einem Gehalt von $1 \mathrm{mg}$ Fe im Liter sehr intensiv und deutlich, selbst bei $1 / 10 \mathrm{mg}$ noch schwach rosa und unter günstigen Umständen selbst bei $1 / 15 \mathrm{mg}$ noch, allerdings nur sehr schwach, bemerkbar ist.

Für das Gelingen der Reaktion sind jedoch farner noch folgende Punkte zu beachten:

Das eisenhaltige Wasser mufs, wie bereits erwähnt, neutral oder schwach alkalisch sein, denn freie Süuren heben die Fürbung anf und starke freie Alkalien geben mit Tannin schon allein starke Färbungen; diese Färbungen sind freilich anderer Art, als die durch Eisensalz hervorgerufenen, sind schmutzig und unschön, und treten auch erst allmälig ein. Doch ist zu bemerken, dafs von Ätznatron und Ätzkali sehon sehr geringe Mengen genügen, um mit Gerbsäure Färbung zu geben, bei Ammoniak und kohlensauren Alkalien tritt sie erst bei gröfserer Menge und nach längerer Zeit ein. Ja, pyrophosphorsaures Natrium in konzentrierter Lösung bewirkt (offenbar 
F. Ger'nard, Bestimmung des Eisens im Brunnenwasser etc. 70

intolge seiner basischen Natur) bei längerem Stehen schon allein eine Färbung der gerbsäurehaltigen Flüssigkeit. In nicht ganz schwachen Lïsungen von Kalk-, Magnesiasalzen etc., ja selbst in konzentrierten Alkalisalzlösungen ruft Gerbsüure bei längerem Stehen Trübungen hervor, die störend wirken könnten.

Es ist nun aber sehr leicht, diese Fehlerquellen zu vermeiden, und man arbeitet nach folgender Methode absolnt sicher:

Man hiilt folgende Flüssigkeiten vorrätig:

1. Eine Jüsung von 1 Teil kryst. Natriumpyrophosphat in 20 Teilen Wasser,

2. Eine I,ismung von 1 Teil Tannin in 20 Toilen schwachem Spiritus.

3. Kine Eisenlïsung, die in jedem $\mathrm{ccm} 0,1 \mathrm{mg}$. Fe in der Form von Eisen-Natriumpyrophosphat enthiilt.

Man erhält solche Flüssigkeit, wenn man 0,898 Eisenalaun und 2,5 kryst. Natriumpjrophosphat in Wasser löst und zum Ijiter verdünnt, oder indem man $1 \mathrm{~g}$ offizinelle Eisenchloridflüssigkeit mit $50 \mathrm{ccm}$ der obigen Natriumpyrophosphatlösung mischt und die klare Flüssigkeit ebenfalls auf 1 Liter bringt. Diese Lösungen sind ohne Zersetzung lange Zeit baltbar, bleiben klar und scheiden keine basischen Salze aus.

Wird das zu untersuchende Wasser ganz frisch eingeliefert, wo es noch klar ist und das Eisen sich noch als Karbonat in gelöstem Zustande befindet, so versetzt man $100 \mathrm{ccm}$ desselben sofort mit $20 \mathrm{ccm}$ der Lösung von pyrophosphors. Natrium und sodann mit 5 Tropfen Tanninlösung. $\quad(20 \mathrm{ccm}$ Natriumpyrophosphatlösung dürften wohl genügen, selbst im härtesten Brunnenwasser. das anfänglich sich ausscheidende Calciumpyrophosphat wieder zu lösen).

Nach wenigen Sekunden ist die erwünschte konstante Färbung eingetreten und diese vergleicht man in bekannter Weise mit der Fürbung, die eintritt, wenn man $100 \mathrm{ccm}$ eisenfreien Wassers mit $20 \mathrm{ccm}$ Natriumpyrophosphat und einer gewissen Menge Eisenlösung und Tannin versetzt.

Etwas umständlicher ist der einzuschlagende Weg, wenn (wie es wohl meistens der Fall ist) sich das Fisen bereits ganz oder zum Teil abgeschieden hat. 
Dann schüttelt man das Wasser auf, bringt etwa $150 \mathrm{ccm}$ in eine Kochflasche, fügt 1,0 Oxalsäure hinzu und erhitzt. Der Eisenniederschlag löst sich in der Oxalsäure sehr leicht. Die noch heifse Flüssigkeit versetzt man nun mit einem Körnchen Kaliumcitrat (um eine Fällung des Eisens zu verhindern) und darauf mit so viel reinem Calciumkarbonat, dals die Oxalsäure vollständig gesättigt wird und eine neutrale Flüssigkeit entsteht. Dieselbe wird klar abfiltriert, was schnell und leicht gelingt und ist nun vortrefflich geeignet zur Anstellung der Tanninreaktion.

Man fügt zunächst wieder $20 \mathrm{ccm}$ Natriumpyrophosphat, damn 5 Tropten Tanninlösung zu und verfährt wie oben. Anch diese Prüfung. ist in kurzer Zeit und leicht in durchaus sicherer Weise zu erledigen.

Als Vergleichsfliussigkeit verwendet man am besten eine Flüssigkeit, die man erhält durch Vermischung von $100 \mathrm{cem}$ Wasser mit $20 \mathrm{ccm}$ Natriumpyrophosphat, 5 Tropfen Tannin und 1 com der obigen Eisenlösung. Diese Flüssigkeit enthiilt $0,1 \mathrm{mg} \mathrm{Fe}$, entspricht, also $1 \mathrm{mg}$ Fe im Iiter. Starkere Fürbungen oder schwächere scheinen mir weniger geeignet zur Vergleichung.

Am schnellsten und sichersten lsommt man, wie gresagt, zum Ziele, wenn das Wasser frisch und noch klar eingeliefert wird.

Wenn das Eisen sich bereits ausgeeschieden hat, so kann man leicht getäuscht werden: man kaun nicht wissen, ob das Wasser vorher ganz klar gewesen ist, ob die Truibung ausschliefslich von ausgeschiedenen Eisenhydroxyd herrührt, oder ganz oder zun Teil von Eisenrost, der nicht gelöst gewesen war, oder auch von eisenhaltiger Erde oder dergl. Fs handelt sich bei der Untersuchung meist um Wasser aus frisch erbohrten Brunnen, wo dergleichen Vorkommnisse besonders leicht möglich sind.

Der Chemiker ist meist nicht im Stande, zu entscheiden, ob der schmutzige Niederschlag von gelöst gewesenem und wieder ausgeschiedenem Eisen herrührt, oder andern Ursachen zuzuschreiben ist, und daher wird er in allen Fällen gut thun, wenn es irgend mïglich jst, sich ganz frisch geschöpttes, noch klares Wasser bringen zu lassen. Die Untersuchung führt dann zu ganz sicheren Resultaten und ist anf die leichtoste und schnellste Weise auszuführen. 